\title{
Clinicohematological Characteristics and Survival Analysis of Aplastic Anemia in Pakistan; A Single Centre Experience
}

\author{
Nida Anwar ${ }^{1, *}$, Aisha Arshad ${ }^{1}$, Naveena Fatima ${ }^{2}$, Sumaira Shaheen², Tahir Sultan Shamsi ${ }^{1}$ \\ ${ }^{1}$ Department of Hematology, National Institute of Blood Disease \& Bone Marrow Transplantation, Karachi, Pakistan. \\ ${ }^{2}$ Department of Research and Development, National Institute of Blood Disease \& Bone Marrow Transplantation, Karachi, Pakistan.
}

\begin{abstract}
Objective: Aplastic anemia (AA) is characterized by pancytopenia and hypocellular bone marrow. Several factors like infections, toxins, chemotherapeutics and radiations are known causes for the suppression of hematopoietic cells which results in bone marrow failure but the exact etiology is unknown. The current study was conducted to assess the baseline demographics, laboratory and clinical characteristics of patients presenting with aplastic anemia and evaluate their cytogenetic profile.
\end{abstract}

Materials and Methods: A retrospective cohort study conducted at National Institute of Blood Diseases and Bone Marrow Transplantation after approval by Institutional Ethics Committee. In this study, AA patients were enrolled from January 2010 to December 2018 . Data collection included demographic, laboratory and clinical characteristics including age, gender, symptoms, treatment, and blood counts. Cytogenetic analysis was performed on bone marrow samples. Data analysis was done by using SPSS version 23.

Results: Based on camitta classification, a total of 362 aplastic anemia patients were enrolled in the study. The frequency of severe aplastic anemia was most common 199(55\%). Median and interquartile range (IQR) age of overall patients was 17(11-26) years, for children and adult population it was 12(9-14) years and 28 (21-43) years respectively. Male predominance was observed i.e.251(69\%). The most common presenting complaint was fever 202(55.8\%). The median and IQR of hemoglobin (Hb) was 7.8(5.8-9.4)g/dl, MCV 90(83-91)fl, total leucocyte count $($ TLC) $2.6(1.9-3.6) \times 109 / 1$, absolute neutrophil count $($ ANC) $0.64(0.27-1.2) \times 109 / 1$ and platelet count $13(5-27) \times 109 / 1$. Bone marrow cytogenetics was done and 76 (67\%) patients were found to have normal karyotype. CMV was positive in 24(6.6\%). Majority of patients were treated with blood transfusion and supportive care only $230(64 \%)$ and the survival rate was $84 \%$.

Conclusion: In conclusion, the study represents a large cohort of aplastic anemia in the country. Majority of cases were acquired aplastic anemia predominantly being severe aplastic. Limited numbers of patients opted for standard treatment options probably due to financial reservations to afford standard treatments like immune suppression therapy and hematopoietic stem cell transplant. Further, local studies with larger number of sample size and provision of standard treatment options are needed to explore the treatment response, etiological factors, prognosis and outcomes.

Keywords: Aplastic anemia, Cytogenetics, Clinicohematological characteristics, Laboratory analysis, Survival outcome, Pakistan.

\section{INTRODUCTION}

Aplastic anemia (AA) is one of the significant non- malignant blood disorder in Pakistan which is characterized by pancytopenia, trilineage bone marrow aplasia without increased reticulin fibrosis [1]. Aplastic anemia may be acquired and immune mediated. Most of the cases are idiopathic but environmental factors play significant role including exposure to drugs, toxins and viral infections [1-3]. An activated cytotoxic T-cells expressing interferon- $\gamma$ and tumor necrosis factor- $\alpha$ were also suggested as contributory factor towards the destruction of hematopoietic stem cells immunologically [4].

A progressive increase in the number of cases has been observed in the occurrence of aplastic anemia especially in Asian sub-continent. An estimation of 2 in 1,000,000 persons per year in western countries has been observed as an annual incidence [5], however, it is relatively higher in the far East

*Address correspondence to this author at the Department of Hematology, National Institute of Blood Disease \& Bone Marrow Transplantation,

Karachi, Pakistan Email: drnidairfan@yahoo.com with 4 to 7 per $1,000,000$ persons per year $[6,7]$ which is $2-3$ times more common as compared to west. Acquired AA most commonly presents between the ages of 15 and 25 years, but there is a second smaller peak incidence after age of 60 years. The signs and symptoms vary from non-severe disease to severe life threatening cytopenias which requires treatment. Allogenic hematopoietic stem cell transplantation (HSCT) is standard of treatment in younger patients. Due to limited provision of the facilities, financial reservations and unavailability of matched donors, HSCT is done for limited number of AA patients in Pakistan. Immune suppression treatment (IST) is one of alternative treatment to HSCT i.e. Antithymocyte globulin (ATG) and cyclosporine treatment. Unfortunately, it is also an expensive treatment option and is beyond the affordability of many patients living in third world country like Pakistan. Thus supportive care i.e. blood product transfusion is usually opted by majority of such patients deferring definitive first line treatment. Nevertheless, on the other hand the miseries of such cohort of patients and their relatives is further increased by difficulty in getting frequent and safe

www.njhsciences.com 
blood and blood products due to scarcity of voluntary blood donation in the country $[8,9]$. There is lack of data on aplastic anemia from Pakistan because of limited number of studies .The present study was conducted aiming to assess the clinicohematological characteristics and cytogenetic profile of disease among Pakistani population.

\section{MATERIALS AND METHODS}

A retrospective cohort study was conducted at National institute of blood diseases and bone marrow transplantation, Karachi Pakistan which is one of the referral centres for diagnosis and management of hematological disorders in the country. All Aplastic anemia patients including pediatric $(<18$ years) and adults ( $\geq 1$ 8years) subjects diagnosed at our centre fulfilling the camitta classification were enrolled in the study.

The study was approved by the Institute's Research Ethics Committee. In this study, 362 adult and pediatric patients with aplastic anemia including (251 males and 111 females) were enrolled during the period from January 2010 to December 2018. Based on camitta classification the patients were divided into three groups, non-severe, severe and very severe aplastic anemia. Suspected and confirmed aplastic anemia patients were traced from wards and follow up hematology clinics. Inclusion of all participants was voluntary. Medical records were reviewed and data collected into an access database. Data collected including demographics, laboratory and clinical measurement including age, gender, blood counts, cytogenetic, viral profile, treatment offered. Status of alive and expire of patients during the study period of 9 years was also recorded.

Blood samples of ten-milliliter were collected from all the subjects and were distributed in different anticoagulants tubes including EDTA, citrate and clot. 3cc blood kept in K2EDTA anticoagulant tube was then used to analyze complete blood picture using an automated hematology Sysmex XN-1000 analyzer. All blood samples were analyzed soon after blood collection. Bone marrow and trephine biopsy results were also recorded.

Cytogenetic analysis was performed on overnight, 24-h un-stimulated and 72-h stimulated bone marrow cultures using standard procedures (to rule out hypoplastic MDS and establish if any chromosomal aberration). Bone marrow and trephine biopsy results were also recorded (to establish diagnosis as per camitta criteria).Viral profile included Anti-HCV, HBsAg, HIV I/II serology performed using chemiluminescence technique (to rule out post viral/post hepatitis aplastic anemia). Cytomegalovirus (CMV), HSV was performed by RT polymerase chain reaction (PCR) and HEV was performed by DIA PROHEV IgM.

\section{STATISTICAL ANALYSIS}

Descriptive analysis was calculated as median, interquartile range (IQR), frequencies and percentages by using statistical package for the social sciences version 23.0 (SPSS Inc, Chicago, IL, USA). Survival analysis was done by using Kaplan-Meier method.

\section{RESULTS}

A total of 362 patients were enrolled in the study. Male to female ratio was 2.2:1. Out of total, 191(53\%) were pediatric subjects and 171(47\%) were adults. The patients were followed up to 9 years ranging from 5-2640 days. The median and interquartile range (IQR) age of overall patients was 17(11-26) years, for children and adult population it was $12(9-14)$ and 28 (21-43) respectively. The frequency of patients according to camitta classification is shown in Fig. (1). The most common presenting complaint was fever 202(55.8\%) followed by weakness 191(52.7\%), gum bleeding $102(28.1 \%)$, epistaxis $58(16 \%)$, bruises $38(10.4 \%)$, shortness of breath 21 (5.8\%) and hepatomegaly 12(3.3\%).

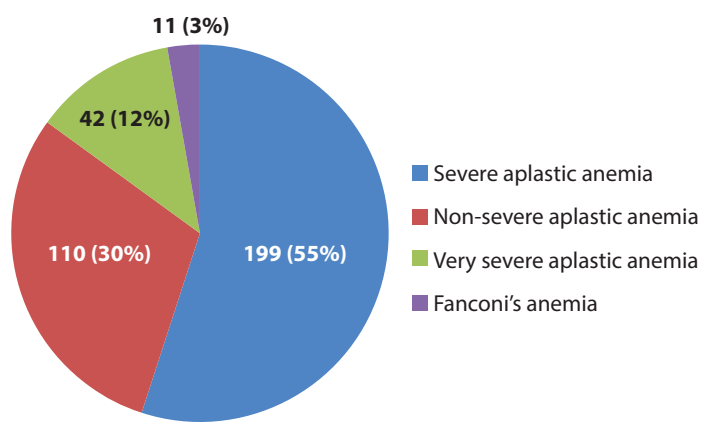

Fig. (1). Frequency of Aplastic Anemia Patients according to Camitta Classification in Percentage $(\mathrm{N}=362)$.

Demographic and hematological parameters included hemoglobin $(\mathrm{Hb})$, total leucocyte count (TLC), absolute neutrophils count (ANC) and platelets counts along with the cytogenetic analysis, depicted in Table $\mathbf{1}$. The positivity of viral infections at baseline was also observed and presented in Table 01 showing high percentage of CMV. Regarding treatment, the majority of patients were treated with blood transfusion and supportive care only $230(64 \%)$ followed by patients treated by HSCT $65(18 \%)$, ATG in combination with cyclosporine $40(11 \%)$ and cyclosporine $27(7 \%)$.

Out of 362 patients, 14 patients were lost to follow up due to financial issues and reluctance to visit from remote areas. The survival analysis was done on 348, out of which 291 patients were alive. The overall survival rate was $84 \%$, observed during the 9 years of study period (Fig. 2). Death rate during 9 years was 57(16\%) with median 180 (8-1560) days. The most common cause of death was sepsis $30(52.6 \%)$ followed by bleeding in $5(8.8 \%)$ patients respectively. However, the 
cause of death could not be ascertained in patients 22(38.6\%).

Table 1. Demographic, Laboratory and Clinical Characteristics of Aplastic Anemia Patients.

\begin{tabular}{|c|c|}
\hline \multicolumn{2}{|c|}{ Total number of patients $\mathbf{~}=\mathbf{3 6 2}$} \\
\hline Age in years, Median(IQR) & $17(11-26)$ \\
\hline Male/female ratio & $2.2: 1$ \\
\hline Hb (g/dl), Median(IQR) & $7.8(5.8-9.4)$ \\
\hline MCV(fl), Median(IQR) & $90(83-91)$ \\
\hline TLC(10^9/1), Median(IQR) & $2.6(1.9-3.6)$ \\
\hline ANC(10^9/1), Median(IQR) & $0.64(0.27-1.2)$ \\
\hline Platelet counts(10^9/1), Median(IQR) & $13(5-27)$ \\
\hline Cytogenetics (n=114) (\%) \\
\hline Normal Karyotype & $76(67 \%)$ \\
\hline Abnormal Karyotype & $38(33 \%)$ \\
\hline Co-morbidities (\%) & \\
\hline Hypertensive & $21(5.8 \%)$ \\
\hline Diabetes mellitus & $20(5.5 \%)$ \\
\hline Viral infections (\%) & $24(6.6)$ \\
\hline CMV & $09(2.4)$ \\
\hline HSV & $03(0.8)$ \\
\hline HCV & $02(0.5)$ \\
\hline HbsAg & $02(0.5)$ \\
\hline HEV &
\end{tabular}

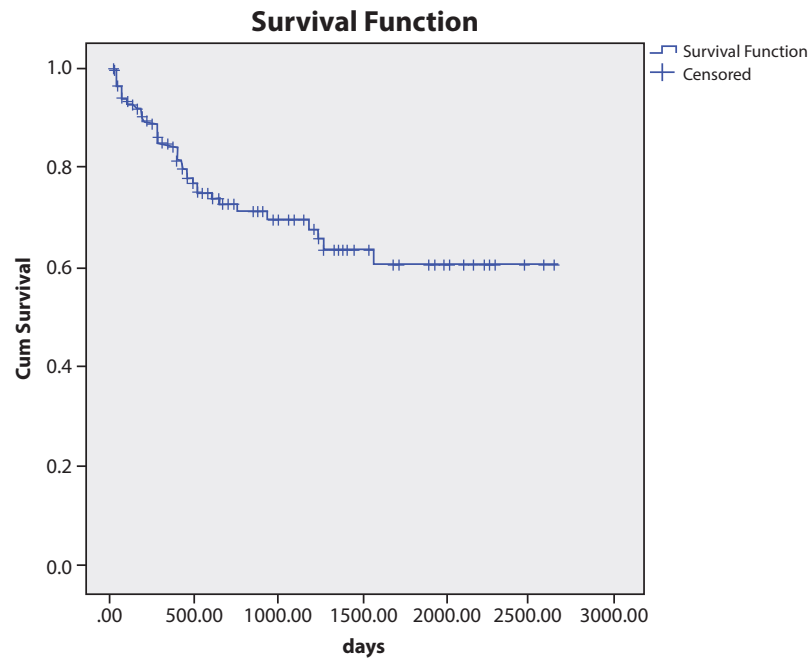

Fig. (2). Survival Analysis of Aplastic Anemia Patients.

\section{DISCUSSION}

Aplastic anemia is an acquired hematological disorder with the disease spectrum of cytopenia (s) and bone marrow failure [10]. Due to complex involvement of environmental, clinical, genetic risk factors the exact etiology is still poorly understood [1]. Several genetic and environmental factors are considered to be the leading cause of the disease and have differentiated disease as inherited and acquired [11]. In the present study, assessment of clinico hematological disease characteristics was carried out for the first time in province of Sindh and second in Pakistan. Severe aplastic anemia (55\%) patients were most prevailing as compared to very severe and non-severe cases classified according to camitta classification which is similar to the findings of Jeong et al. [12] and Akram $\mathrm{Z}$, et al. [1]. However, in another study from Pakistan, Wali et $a l$. found increased number of very severe aplastic anemia patients [13]. The median age of the patients was 17 which is higher as compared to the study of Das et al. [10] and less than the study of Issaragrisil et al. [6]. A total of 212 patients were within the age group of 10-29 years which is similar to the age group patients of Akram Z. et al. [1]. The higher predominance of male patients was observed compared to females with ratio of 2.2:1 which is similar to the studies of [1, 10, 13-15] but dissimilar to the studies [16] as they had observed slightly higher female predominance in their patients. In our study male preponderance might be due to social bias factor as they are considered more important in rural areas and brought preferentially for treatment as discussed in study of Pervez et al. [17] The most common presenting complaint was fever $(55.8 \%)$ and weakness $(52.7 \%)$ which was more or less similar to the previous studies $[10,13,18]$. In Hematological parameters, the median of all three cell lineage was low with $\mathrm{Hb} 7.8 \mathrm{~g} / \mathrm{dl}$, TLC $2.6\left(10^{\wedge} 9 / 1\right)$ and platelet counts $13\left(10^{\wedge} 9 / 1\right)$ and low hemoglobin and platelet counts and TLC as observed in study of Das et al. [10]. Cytogenetic analysis was done in only 114 patients. Normal karyotype was found in (67\%) while abnormal karyotype was observed in (33\%) which is higher as compared to the study of Das et al. and Gupta et al. [10,19].

The clinical relevance of cytogenetic in AA might be helpful in considering treatment regimen. A study reported that patients who had trisomy at diagnosis could get the treatment similar to the patients with normal karyotype. However, one of the study groups proposed that trisomy 8 clone triggers cytotoxic CD8+ T cells which inhibit preferentially trisomy 8 cells and these patients respond to ATG with normalization of $\mathrm{T}$ cells repertoire [20]. Further studies are needed to be done to establish prognostic significance of individual cytogenetic abnormality in AA. Certain cytogenetic abnormalities are specific for MDS and may help to rule out hypoplastic MDS in otherwise AA patients.

In our study, $10.8 \%$ patients were positive for one or more of the virus including positive occurrence of CMV (6.6\%), followed by HSV (2.4\%), HCV $0.8 \%$ which is slightly greater as compared to the positivity of HCV found in a study of Wali and colleagues [13]. 
In third world country like Pakistan, expensive treatment options are not readily affordable by most of the patients. In our study, supportive care and blood transfusion was given to $64 \%$ patients followed by HSCT18\% and IST with cyclosporine only in $11 \%$ which is similar to the study of Wali et al. [13]. The outcome revealed $84.2 \%$ of alive patients and $15.8 \%$ patients who were expired at average of 9.9 months of treatment. Sepsis was the most common cause of death followed by bleeding which is similar to a study [13] reporting infection as the most common cause of death.

\section{CONCLUSION}

The present study is one the first study in the province and second largest in country revealing clinicohematological characteristics of aplastic anemia patients of Pakistan with sizeable number of patients. Majority of cases were acquired including higher number of severe aplastic anemia. To overcome adverse disease implications, standard treatment modality with early diagnosis, close clinical follow up and upfront hematopoietic stem cell transplant (HSCT) must be considered especially in younger population. Immune suppression treatment (IST) must be offered to patients not eligible for HSCT. Further, local studies with larger number of sample size and provision of standard treatment options are needed to explore the treatment response, etiological factors, prognosis and outcomes.

\section{LIST OF ABBREVIATIONS}

AA: Aplastic Anemia.

CMV: Cytomegalovirus.

PCR: Polymerase Chain Reaction.

Hb: Hemoglobin.

TLC: Total Leucocytes Counts.

ANC: Absolute Neutrophils Count.

HSCT: Hematopoietic Stem Cell Transplant.

IST: Immune Suppression Treatment.

ATG: Antithymocyte Globulin.

WBC: White Blood Cells.

RBC: Red Blood Cells.

GTG: G-bands via Trypsin Using Giemsa.

SPSS: Statistical Package for the Social Sciences.

\section{AUTHORS' CONTRIBUTION}

Nida Anwar: Had the main idea of the study, searched the literature and drafted the manuscript.

Aisha Arshad: Contributed in data collection and manuscript writing.

Naveena Fatima: Contributed in data analysis.

Sumaira Shaheen: Contributed in data collection and patient recruitment.

Tahir Sultan Shamsi: Supervised the study and critically reviewed the manuscript.

\section{CONFLICT OF INTEREST}

Declared none.

\section{ACKNOWLEDGEMENTS}

Declared none.

\section{REFERENCES}

[1] Akram Z, Ahmed P, Kajigaya S, et al. Epidemiological, clinical and genetic characterization of aplastic anemia patients in Pakistan. Ann Hematol 2019; 98(2): 301-12. DOI: 10.1007/s00277-018-3542-z

[2] Brodsky RA, Jones RJ. Aplastic anaemia. Lancet 2005; 365 : 1647-56. DOI: 10.1016/S0140-6736(05)66515-4

[3] Bakhshi S, Abella E. Aplastic Anemia. 2009; Available at: http:// emedicine.medscape.com/article/198759-overview [Accessed June 7, 2009].

[4] Morales-Mantilla DE, King KY. The Role of Interferon-gamma in hematopoietic stem cell development, homeostasis, and disease. Curr Stem Cell Rep 2018; 4(3): 264-71. DOI: 10.1007/s40778-018-0139-3

[5] Maluf E, Hamerschlak N, Cavalcanti AB, et al. Incidence and risk factors of aplastic anemia in Latin American countries: the LATIN case-control study. Haematologica 2009; 94(9): 1220-6. DOI: 10.3324/haematol.2008.002642

[6] Issaragrisil S, Kaufman DW, Anderson T, et al. The epidemiology of aplastic anemia in Thailand. Blood 2006; 107(4): 1299-307. DOI: 10.1182/blood-2005-01-0161

[7] Zhu XF, He HL, Wang SQ, et al. Current treatment patterns of aplastic anemia in China: A prospective cohort registry study. Acta Haematol 2019; 142(3): 162-70. DOI: $10.1159 / 000499065$

[8] Hernández-Rivera EG. Hematopoietic stem-cell transplantation in aplastic anemia. Rev Invest Clin 2005; 57 (2): 298-304.

[9] Keohane EM. Acquired aplastic anemia. Clin Lab Sci 2004; 17(3): 165-71.

[10] Das S, Tilak V, Gupta V, Singh A, Kumar M, Rai A. Clinical, hematological, and cytogenetic profile of aplastic anemia. Egypt J Haematol 2015; 40(1): 3-10. DOI: 10.4103/1110-1067.155779

[11] Taj M, Shah T, Aslam SK, et al. Environmental determinants of aplastic anemia in Pakistan: A case-control study. Z Gesundh Wiss 2016; 24(5): 453-60. DOI: 10.1007/s10389-016-0743-6

[12] Jeong DC, Chung NG, Kang HJ, et al. Epidemiology and clinical long-term outcome of childhood aplastic anemia in Korea for 15 years: Retrospective study of the Korean Society of Pediatric Hematology Oncology (KSPHO). J Pediatr Hematol Oncol 2011; 33(3): 172-8. DOI: 


\subsection{7/MPH.0b013e31820826a8}

[13] Wali R, Fidoo Z, Adil S, Naqvi MA. Aplastic Anemia. Clinicohaematological features, treatment and outcome analysis. JCPSP 2011; 21(4): 219-22.

[14] Biswajit H, Pratim PP, Kumar ST, Shilpi S, Krishna GB, Aditi A. Aplastic anemia: A common hematological abnormality among peripheral pancytopenia. N Am J Med Sci 2012; 4(9): 384-8. DOI: 10.4103/1947-2714.100980

[15] Gupta V, Tripathi S, Singh T, Tilak V, Bhatia B. A study of bone marrow failure syndrome in children. Ind J Med Sci 2008; 62(1): 13. DOI: 10.4103/0019-5359.38917

[16] Vaht K, Göransson M, Carlson K, et al. Incidence and outcome of acquired aplastic anemia: real-world data from patients diagnosed in Sweden from 2000-2011. Haematologi- ca $2017 ; 102(10): \quad 1683-90$. DOI: $10.3324 /$ haematol.2017.169862

[17] Ahmed P, Chaudhry QU, Satti TM, et al. Epidemiology of aplastic anemia: A study of 1324 cases. Hematology 2020; 25(1): 48-54. DOI: 10.1080/16078454.2019.1711344

[18] Yarrnal PJ, Basu D. Haematological profile of aplastic anemia in childhood. Ind J Hemat Blood Transf 2003; XXI: 107-9.

[19] Gupta V, Kumar A, Saini I, Saxena AK. Cytogenetic profile of aplastic anaemia in Indian children. Ind J Med Res 2013; 137: 502-6.

[20] Gupta V, Brooker C, Tooze JA, et al. Clinical relevance of cytogenetic abnormalities at diagnosis of acquired aplastic anaemia in adults. Br J Haematol 2006; 134(1): 95-9. DOI: 10.1111/j.1365-2141.2006.06105.x 